\title{
Revisiting the Post-reionization Kinetic Sunyaev-Zel'dovich Effect on the Cosmic Microwave Background Fluctuations
}

\author{
H. Park*, E. Komatsu, P. R. Shapiro \\ Univrsity of Texas at Austin, U.S.A. \\ E-mail: hcosmosbeastro.as.utexas. edu, komatsu@astro.as.utexas.edu, \\ shapirodastro.as.utexas.edu
}

\begin{abstract}
In order to study the reionization contribution to the kinetic Sunyaev-Zel'dovich effect, we need to subtract the post-reionization contribution to the observed total signal. In this poster, we show post-reionization calculation of the kinetic Sunyaev-Zel'dovich (kSZ) effect required to acquire the reionization contribution for our subsequent study. In the poster, we present the $3 \mathrm{rd}$ order perturbation theory (3PT) as an effective approximation for calculating the post-reionization kSZ effect. We find that, at $l=3000 \sim 7000$, about $60 \%$ of the contribution comes from large scale $(k<1.5 h / M p c)$ structure at high redshifts $(z>1)$ where the 3PT is valid. Future work should model the reionization contribution so that the observed data from currently ongoing surveys can be used to constrain the reionization history.
\end{abstract}

Frank N. Bash Symposium New Horizons In Astronomy,

October 9-11, 2011

Austin Texas

${ }^{*}$ Speaker. 


\section{Introduction}

New experiments are underway to measure the temperature fluctuations of the cosmic microwave background (CMB) on small angular scales caused by the Compton scattering of $\mathrm{CMB}$ photons by free electrons in the intergalactic medium, the Sunyaev-Zel'dovich (SZ) effect. Inside galaxy clusters, low-energy CMB photons were up-scattered by hot intergalactic electrons because of their microscopic thermal velocities (thermal SZ, tSZ). Outside clusters, free electrons with bulk motions that departed from pure Hubble flow also scattered CMB photons (kinetic SZ, kSZ), first during the epoch of reionization (EOR), when light from the first galaxies created giant patches of ionized gas, and later, after these patches had grown and merged to create a fully-ionized universe. The tSZ signal is strongest, but can be separated from the others by its unique frequency dependence, with a null at $\sim 220 \mathrm{GHz}$. The post-reionization $\mathrm{kSZ}$ signals can be modeled with full ionization assumption. After subtracting the post-reionization $\mathrm{kSZ}$ signals to the total signal, we are left with the EOR contribution that contains information about the history of the first stars and galaxy formation that led to reionization.

In the poster, we revisit the post-reionization kSZ calculation and show the 3PT as an effective approximation for the calculation. We assume that the gas follows the total matter distribution at the scale of interest.

\section{Formalism of the Post-reionization kSZ Effect}

The change of the CMB temperature due to the kSZ effect is given by integration of the Doppler shift of the scattered photons along the line of sight $(\hat{\gamma})$

$$
\frac{\Delta T}{T}(\hat{\gamma})=\int d l e^{-\tau} \bar{n}_{e} \sigma_{T} \hat{\gamma} \cdot X \mathbf{v}(1+\delta),
$$

where $n_{e}, \sigma_{T}$ and $d l$ are number density of electrons, Thomson scattering cross-section and differential element of the physical distance, that are multiplied togerther to give the fraction of scattered photons as the light proceeds by $d l . e^{-\tau}$ with $\tau \equiv \int_{0}^{l} d l \sigma_{T} n_{e}$ expresses the attenuation of the signal due to the optical depth from oberserver to the medium where the signal is generated. Throughout the poster, we express the velocity in units of the speed of light. Therefore, by $\mathbf{v}$, we actually mean $\mathbf{v} / c . X$ denotes the mean ionized fraction of the universe. The resulting CMB power spectrum from the $\mathrm{kSZ}$ effect is

$$
C_{l}=\bar{n}_{e, 0}^{2} \sigma_{T}^{2} \int \frac{d x}{x^{2}}(1+z)^{4} e^{-2 \tau} P_{X q \cdot \hat{\gamma}}\left(\frac{l}{x}, z\right),
$$

where $\mathbf{q} \equiv \mathbf{v}(1+\delta), \bar{n}_{e, 0} \equiv \bar{n}_{e} /(1+z)^{3}$ and $x$ is the comoving distance from the observer to the medium. Hence, the key part in the estimation of the kSZ effect is to model the power spcetrum of the ionized line of sight momentum field, $P_{X q \cdot \hat{\gamma}}(k, z)$.

For the post-reionization contribution of the kSZ effect, we can assume a fully ionized universe, i.e., $X=1$, and only have to model the density and velocity field. If we assume the density and velocity field to be completely linear, $P_{X q \cdot \hat{\gamma}}=P_{q \cdot \hat{\gamma}}$ reduces to a second order expression as following $[1,2]$.

$$
P_{q \cdot \hat{\gamma}}(k, z)=\frac{\dot{a}^{2} f^{2}}{2} \int \frac{d^{3} k^{\prime}}{(2 \pi)^{3}} P_{\delta \delta}\left(\left|\mathbf{k}-\mathbf{k}^{\prime}\right|, z\right) P_{\delta \delta}\left(k^{\prime}, z\right) \frac{k\left(k-2 k^{\prime} \mu^{\prime}\right)\left(1-\mu^{\prime 2}\right)}{k^{\prime 2}\left(k^{2}+k^{\prime}-2 k k^{\prime} \mu^{\prime}\right)}
$$


In non-linear regime, [3] derived a non-linear expression assuming that the velocity field does not have vorticity and therefore the Fourier compononent of velocity is still parallel to the direction of the mode $(\mathbf{v} \propto \hat{\mathbf{k}})$. This is a good approxmiation since the gravity would not generate the vorticity in the velocity field unless we look at an extemely non-linear regime that would not be important for the kSZ effect. Under those assumptions, the power spectrum of the momentum field is given as following.

$$
P_{q \cdot \hat{\gamma}}(k, z)=\frac{1}{2} \int \frac{d^{3} k^{\prime}}{(2 \pi)^{3}}\left(1-\mu^{\prime 2}\right)\left(P_{\delta \delta}\left(\left|\mathbf{k}-\mathbf{k}^{\prime}\right|\right) P_{v v}\left(k^{\prime}\right)-\frac{k^{\prime}}{\left|\mathbf{k}-\mathbf{k}^{\prime}\right|} P_{\delta v}\left(\left|\mathbf{k}-\mathbf{k}^{\prime}\right|\right) P_{\delta v}\left(k^{\prime}\right)\right)
$$

\section{Modeling the Post-reionization kSZ Effect}

In principle, when we evaluate Eq.2.4 with precise power spectra $\left(P_{\delta \delta}, P_{v v}, P_{\delta v}\right)$, we obtain the line of sight momentum field and compute the kSZ signal from post-reionization regime precisely. In reality, computing the power spectra down to small enough scales requires large-scale and high-resolution simulations, and is too expensive to be done for different cosmological parameters. Therefore, one has to use an approximate way to compute the line-of-sight momentum power spectrum quickly.

[4] substituted a non-linear density power spectrum from the halo fitting model of [5] to the linear expression of the momentum power spectrum (Eq. 2.3) after justifying it by comparing it with their simulation. In this report, we use power spectra from the 3rd order perturbation theory with the non-linear expression (Eq. 2.4).

\subsection{The 3rd Order Perturabtion Theory}

Keeping the next-to-leading order terms in the cosmological equations for density and velocity of the medium enables us to extend the analytic solution to the non-linear regime [8]. The 3rd order perturabtion theory power spectrum is known to agree at $10 \%$ level at $z>1.5$ and $k<1(\mathrm{~h} / \mathrm{Mpc})$ [6]. We investigate the validity of the 3PT in terms of calculating the kSZ signal. In this study, we adobt the WMAP7 cosmology parameters given by $\Omega_{\Lambda}=0.728, \Omega_{m}=0.272, \Omega_{b}=0.0455$, $h=0.704, \sigma_{8}=0.81, n_{s}=0.967$ and $z_{o v}=6[7]$.

\section{Result}

We first investigate the validity of the 3PT by comparing the $C_{l}$ calculated from our formalism and another one by [4] (See figure 1). Our result shows a reasonable agreement with that of [4] within $10 \%$ between $l=3000$ and $l=10000$, which is being probed by current experiments. We also compare the contribution from where the 3PT is valid and where the 3PT is not valid by looking into the integrand (See Figure 2). We tabulated the relative ratio of the contribution from where the 3PT is valid to the total integral at different $l$ 's (See Table 1). We find that a significant contribution comes from the regime where the 3PT is valid.

\section{Discussion and Future Work}

In this report, we showed that the 3PT is useful for calculating the non-linear post-reionization $\mathrm{kSZ}$ effect in the range of the multipoles in which the $\mathrm{kSZ}$ signal is expected to be measured. About 


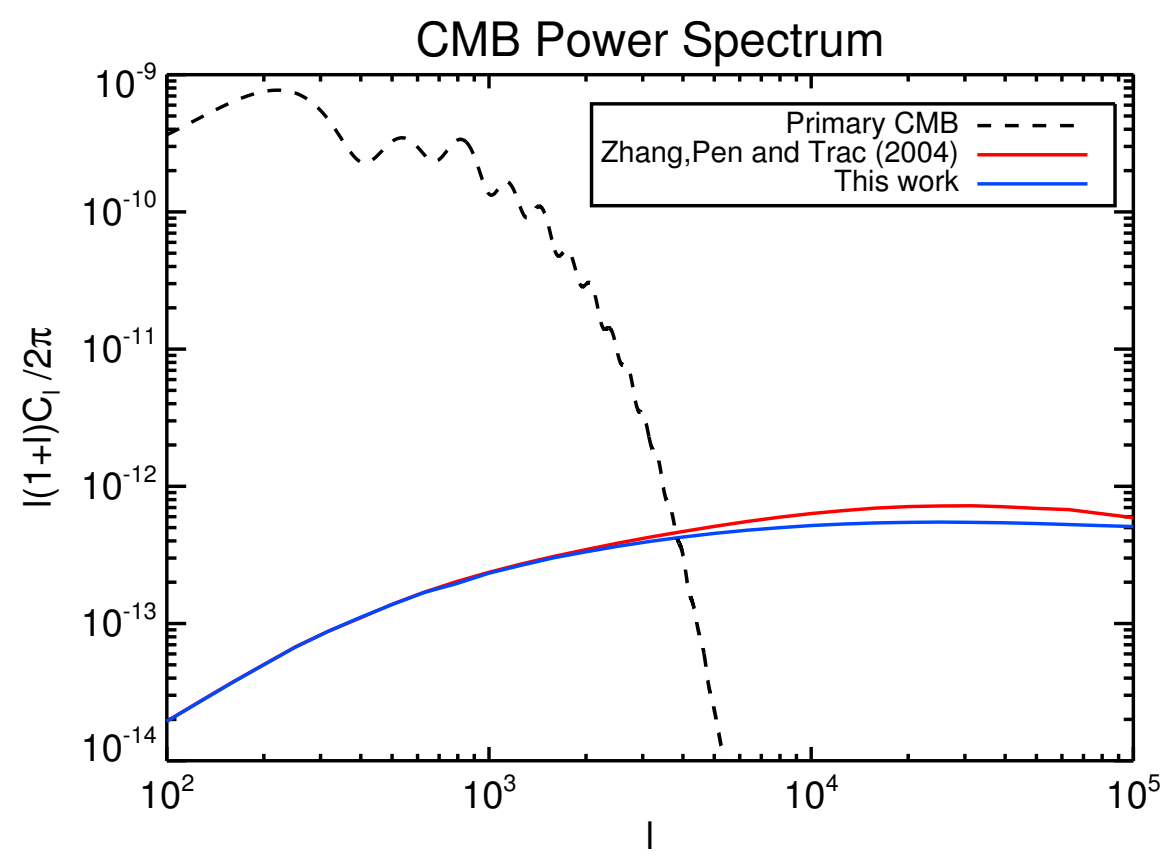

Figure 1: The power spectrum of the CMB and the kSZ effect. The dotted line shows the power in the limit of the linear theory. The blue solid line is the kSZ signal calculated from our 3PT formalism and the red line is calculated from the formalism of [4]. The primary CMB (dashed black line) is also plotted for comparison.

Table 1: The ratio of $z>1$ contribution of $C_{l}$ to the total value

\begin{tabular}{|l|l|}
\hline Multipole moment $(l)$ & $z>1$ contribution \\
\hline 100 & $29 \%$ \\
\hline 300 & $54 \%$ \\
\hline 1000 & $61 \%$ \\
\hline 3000 & $60 \%$ \\
\hline 5000 & $60 \%$ \\
\hline 10000 & $61 \%$ \\
\hline
\end{tabular}

$60 \%$ of the total signal is contributed by the wave number range where the 3PT is accurate enough. We obtain a power spectrum similar to that obtained with the method used by [4].

Our ultimate goal for the subsequent study is to model the full kSZ effect significantly contributed by the reionization epoch. We will study the reionization kSZ effect using our reionization simulation and construct a template that can be used to compare with current experiments like the South Pole Telescope and the Acatama Cosmology Telescope. We will focus on simulating the "patchy" signal that is thought to be the dominant factor of the reionization contribution by post-processing our n-body simulation data with radiative transfer. 


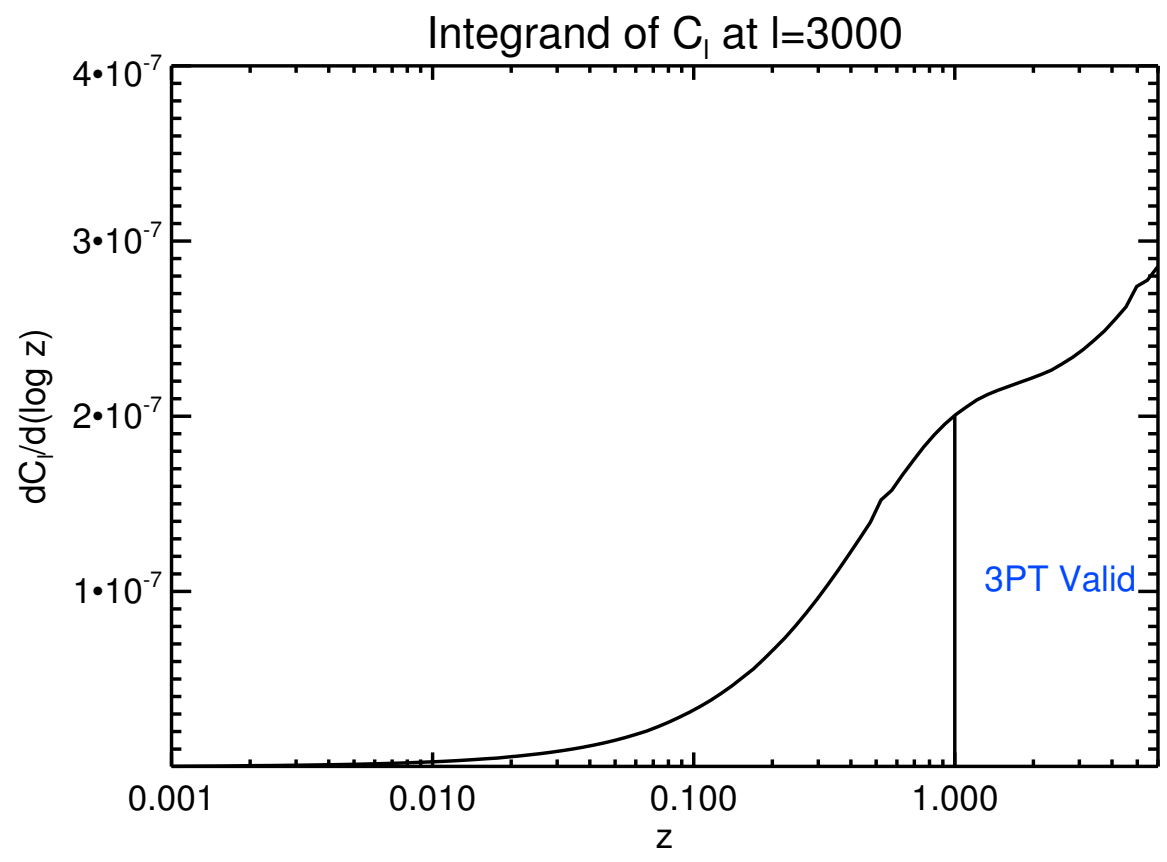

Figure 2: Integrand of $C_{l}$ at $l=3000$. The label is chosen so that the area under the curve gives the total integrated value of $C_{l}$. The right side of the vertical line marks where the 3PT is valid.

\section{References}

[1] Ostriker, J. P., Vishniac, E. T., Generation of microwave background fluctuations from nonlinear perturbations at the ERA of galaxy formation Universe, 1986, ApJ, 306L, 510

[2] Vishniac, E. T., Reionization and small-scale fluctuations in the microwave background, 1987, ApJ, $322,597 \mathrm{~V}$

[3] Vishniac, E. T., Nonlinear Kinetic Sunyaev-Zeldovich Effect, 1987, Physical Review Letters, vol. 88, Issue 21, id. 211301

[4] Zhang, P, Pen, U. L. Trac, H., Precision era of the kinetic Sunyaev-Zel'dovich effect: simulations, analytical models and observations and the power to constrain reionization, 2004, MNRAS, 347, $1224 Z$

[5] Smith, R. E., Peacock, J. A., Jenkins, A., White, S. D. M., Frenk, C. S., Pearce, F. R., Thomas, P. A., Efstathiou, G., Couchman, H. M. P., Stable clustering, the halo model and non-linear cosmological power spectra, 2003, MNRAS, 341, 1311S

[6] Jeong, D., Komatsu, E. , Perturbation Theory Reloaded: Analytical Calculation of Nonlinearity in Baryonic Oscillations in the Real-Space Matter Power Spectrum, 2006, ApJ, 651, 619J

[7] Komatsu, E., Smith, K. M., Dunkley, J., Bennett, C. L., Gold, B., Hinshaw, G., Jarosik, N., Larson, D., Nolta, M. R., Page, L., Spergel, D. N., Halpern, M., Hill, R. S., Kogut, A., Limon, M., Meyer, S. S., Odegard, N., Tucker, G. S., Weiland, J. L., Wollack, E., Wright, E. L., Seven-year Wilkinson Microwave Anisotropy Probe (WMAP) Observations: Cosmological Interpretation, 2011, ApJS, 192, $18 \mathrm{~K}$ 
[8] Vishniac, E. T., Why weakly non-linear effects are small in a zero-pressure cosmology, 1983, MNRAS, 203, 345V 